\title{
Addendum: A dynamic nomenclature proposal for SARS-CoV-2 lineages to assist genomic epidemiology
}

Andrew Rambaut (D), Edward C. Holmes (ID, Áine O'Toole (D), Verity Hill, John T. McCrone, Christopher Ruis, Louis du Plessis and Oliver G. Pybus D

Addendum to: Nature Microbiology https://doi.org/10.1038/s41564-020-0770-5, published online 15 July 2020.

Here we provide clarification regarding the name of the dynamic nomenclature system for SARS-CoV-2 lineages published in our Article. Our nomenclature system should be referred to as the 'Pango' nomenclature system (from the first-person Latin verb meaning 'I set', 'I fix' or 'I record'). It should not be referred to as the 'Rambaut et al.' or 'Pangolin' nomenclature system. Lineages defined by this nomenclature system should be referred to as 'Pango lineages'.

We emphasize that the Pango nomenclature is distinct from, and independent of, any algorithms that are used to implement the system. A growing set of computational tools for working with the Pango nomenclature are provided at https://cov-lineages.org/ and the corresponding GitHub repository, https://github.com/cov-lineages. One popular tool hosted at https://cov-lineages.org/ for classifying SARS-CoV-2 genome sequences is named Pangolin (an acronym for Phylogenetic Assignment of Named Global Outbreak LINeages). The term 'Pangolin lineage' in particular should be avoided because it may be misinterpreted as referring to viral host species, rather than to lineages defined by the Pango nomenclature system.

Published online: 29 January 2021

https://doi.org/10.1038/s41564-021-00872-5

(C) The Author(s), under exclusive licence to Springer Nature Limited 2021 